\title{
Metabolism of 28-Homodolichosterone in Phaseolus vulgaris
}

\author{
Sang Cheul Lee, Se-Hwan Joo, Seung-Hyun Son, Ji Hyun Youn, and Seong-Ki Kim* \\ Department of Life Science, Chung-Ang University, Seoul 156-756, Korea. "E-mail: skkimbio@cau.ac.kr \\ Received October 13, 2010, Accepted November 24, 2010
}

Key Words: Brassinosteroids, Biosynthetic connection, Metabolism of homodolichosterone, Phaseolus vulgaris

\begin{abstract}
The brassinosteroids (BRs) are hormonal chemical signals that are required for normal growth and differentiation in plants. ${ }^{1,2}$ Thus far, more than 50 BRs have been identified from various plant materials, distributed throughout the entire plant kingdom. ${ }^{2-3}$ Among the plants tested, the immature seed of Phaseolus vulgaris is one of the richest plant materials; 22 free BRs and 2 BR conjugates have already been characterized from this species. ${ }^{2,4}$ Recently, we demonstrated that multiple biosynthetic pathways function in the synthesis of endogenous BRs in plants, and further, that these pathways are biosynthetically connected, enabling them to generate bioactive BR. This implies that multiple biosynthetic pathways are also functional and interconnected in P. vulgaris. To confirm this possibility, the in vitro conversion of a $\mathrm{C}_{29}$-BR, 28-homodolichosterone, using a crude enzyme solution prepared from young seedlings of $P$. vulgaris was conducted herein.

It has been demonstrated previously that $P$. vulgaris harbors 6-deoxo-28-homodolichosterone (Fig. 1, 6-deoxo-HDS), a direct biosynthetic precursor of 28-homodolichosterone (HDS). ${ }^{2,4}$ In an effort to confirm the biosynthesis of HDS from 6-deoxoHDS, the in vitro conversion of 6-deoxo-HDS to HDS was assessed. To that end, $P$. vulgaris seedlings ( $5 \mathrm{~g}$ ) were homogenized in a mortar and pestle with cold $0.1 \mathrm{M}$ sodium phosphate ( $\mathrm{pH}$ 7.4). The homogenates were then centrifuged for 30 $\min$ at $20,000 \times \mathrm{g}$. The resultant supernatant was then precipitated via the addition of cold acetone to a final concentration of $40 \%(\mathrm{v} / \mathrm{v})$. The supernatant-acetone mixture was maintained for $10 \mathrm{~min}$ at $-20^{\circ} \mathrm{C}$, and then centrifuged for an additional 10 $\min$ at $13,000 \times \mathrm{g}$. The resultant precipitate was then dissolved in assay buffer (0.1 M sodium phosphate, $\mathrm{pH} 7.4)$.
\end{abstract}
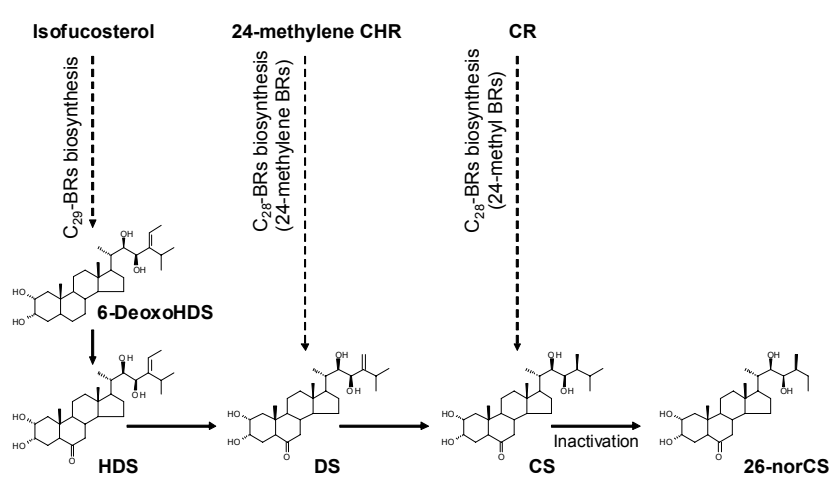

Figure 1. A proposed biosynthetic connection of $\mathrm{C}_{29^{-}}$and $\mathrm{C}_{28}$ - $\mathrm{BRs}$ biosynthesis in $P$. vulgaris. The dotted arrows indicate multiple steps. The solid arrows indicate a single biosynthetic step which is verified in this study.
The reactions were initiated via the addition of 6-deoxoHDS, and incubated for $30 \mathrm{~min}$ at $37{ }^{\circ} \mathrm{C}$. The metabolites of the enzyme reactions were extracted with ethyl acetate $(1.2 \mathrm{~mL})$. The ethyl acetate-soluble fraction was then loaded onto a $\mathrm{C}_{18}$ Sep-Pak cartridge column, and sequentially washed in aqueous $50 \%$ and $60 \% \mathrm{MeOH}$ ( $5 \mathrm{~mL}$ each). The fraction eluted with $100 \% \mathrm{MeOH}$ was concentrated in vacuo, dissolved in $50 \mathrm{uL}$ of $\mathrm{MeOH}$, and subjected to reversed-phase HPLC (Senshu-Pak $\mathrm{C}_{18}, 10 \times 150 \mathrm{~mm}$ ) eluted with aqueous methanol as a mobile phase (0 - $20 \mathrm{~min}$ : 45\%, 20 - 40 min: gradient to $100 \%, 40$ - 60 min: $100 \% \mathrm{MeCN}$ ) at a flow rate of $2.5 \mathrm{~mL} / \mathrm{min}$. Fractions were collected every min, and the HPLC fractions corresponding to HDS (18 - $20 \mathrm{~min})$ were collected. Finally, the enzyme products were derivatized with methaneboronate in pyridine $(1 \mathrm{mg} / 1 \mathrm{~mL})$ and analyzed via GC-MS, using a Hewlett-Packard 5973 mass spectrometer coupled to a 6890 gas chromatograph fitted with a fused silica capillary column (HP-5, $0.25 \mathrm{~mm} \times$ $30 \mathrm{~m}, 0.25 \mu \mathrm{m}$ film thickness).

As summarized in Table 1, the bismethaneboronate (BMB) of the active principal of HPLC fraction 18 - 20 yielded a molecular ion at $m / z 524$, and the prominent ions 481, 411, 387, 370, $357,327,287,167,138$ and 123. The mass spectrum and GC retention time at $37.33 \mathrm{~min}$ were identical to those of authentic HDS BMB, indicating that the active principal in the HPLC fractions was HDS. This confirms that 28 -homodolichosterone can be generated from 6-deoxo-HDS via C-6 oxidation in Phaseolus plants.

Next, the metabolism of HDS $(10 \mu \mathrm{g})$ was assessed via in vitro enzymatic conversion using the same enzyme solution prepared from the $P$. vulgaris seedlings. After $30 \mathrm{~min}$ of assay at $37{ }^{\circ} \mathrm{C}$, the enzyme products were purified by the previously described methods. In the rice lamina inclination assay, biological activities were assessed in fractions 14-17, 19, 20, and 22-24. The active compound in the fractions was derivatized to a BMB, and then analyzed via GC-MS. In HPLC fractions 14 and 15 , the BMB of the compound evidenced the same mass spectrum and GC retention time as those of synthetic dolichosterone (DS) BMB (Table 1), thereby demonstrating that the compound was DS. In HPLC fractions 16 and 17, as a BMB, the obtained mass spectrum of the active compound was identical to authentic 26-norcastasterone (26-norCS) BMB at an identical GC retention time, thus identifying the compound as 26-norCS. HPLC fractions 19 and 20 corresponded to HDS, which was used as a substrate. As a BMB, the compound in HPLC fractions 22-24 yielded an equal mass spectrum at the same GC retention time to that of castasterone (CS) BMB. Therefore, the compound in the fraction was characterized as CS. As a con- 
Table 1. GC-MS analysis for identification of products assayed by a crude enzyme solution prepared from P. vulgaris.

\begin{tabular}{|c|c|c|c|}
\hline \multicolumn{2}{|l|}{ Compound $^{a}$} & \multicolumn{2}{|c|}{$\mathrm{Rt}^{b}$ on GC Prominent ions (relative intensity, \%) } \\
\hline Substrate & Product & & \\
\hline \multicolumn{2}{|c|}{ 6-DeoxoHDS HDS } & 35.035 & $524(\mathrm{M}+, 11), 481(100), 411(11), 387(15), 370(16), 357(14), 327$ (33), 287 (5), $167(44), 138(52), 123(15)$ \\
\hline \multirow[t]{3}{*}{ HDS } & DS & 32.105 & $510(\mathrm{M}+, 26), 495$ (15), 411 (15), 387 (17), 355/356 (20), 327 (97), 287 (10), 153 (72),124 (100) \\
\hline & $\mathrm{CS}$ & 31.105 & $512(\mathrm{M}+, 74), 441(21), 399(17), 358(31), 327$ (9), $287(33), 155(100)$ \\
\hline & 26-norCS & 27.054 & $498(\mathrm{M}+, 100), 483(7), 399(9), 358(26), 328(14), 287(46), 141$ (93) \\
\hline \multirow[t]{2}{*}{ DS } & $\mathrm{CS}$ & 31.105 & $512(\mathrm{M}+, 75), 441(19), 399(16), 358(30), 327$ (12), $287(33), 155(100)$ \\
\hline & 26-norCS & 27.054 & $498(\mathrm{M}+, 100), 483$ (5), 399 (12), 358 (26), 328 (13), 287 (46), 141 (91) \\
\hline $\mathrm{CS}$ & 26-norCS & 27.054 & $498(\mathrm{M}+, 100), 483(8), 399$ (9), 358 (27), 328 (10), 287 (42), 141 (91) \\
\hline \multicolumn{2}{|c|}{ Authentic HDS } & 35.035 & $524(\mathrm{M}+, 10), 481(100), 411(10), 387$ (17), 370 (15), $357(16), 327$ (36), $287(5), 167(47), 138(50), 123(13)$ \\
\hline \multicolumn{2}{|c|}{ Authentic DS } & 32.105 & $510(\mathrm{M}+, 27), 495$ (14), $411(15), 387$ (16), 355/356 (18), 327 (98), 287 (9), 153 (72), $124(100)$ \\
\hline \multicolumn{2}{|c|}{ Authentic CS } & 31.105 & $512(\mathrm{M}+, 78), 441(19), 399(17), 358(30), 327(11), 287(34), 155(100)$ \\
\hline \multicolumn{2}{|c|}{ Authentic 26-norCS } & 27.054 & $498(\mathrm{M}+, 100), 483(8), 399(10), 358$ (27), 328 (11), 287 (42), $141(93)$ \\
\hline
\end{tabular}

${ }^{a}$ Compound was analyzed as a bismethanemoronate (BMB). ${ }^{b}$ Rt: retention time (min)

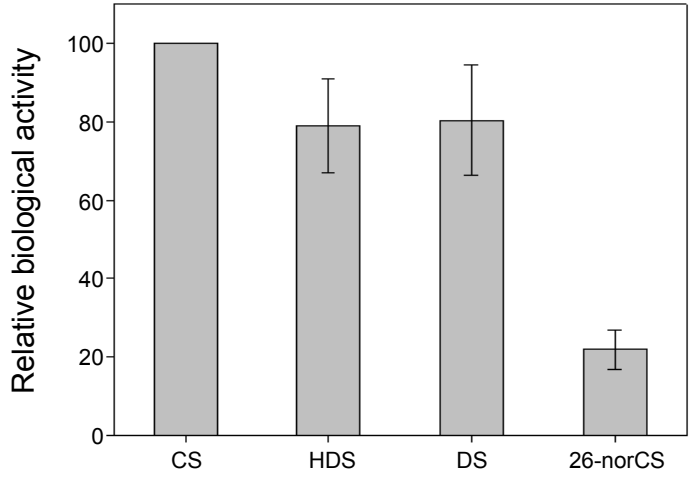

Figure 2. Relative biological activity for CS, DS, HDS and 26-norCS in the rice lamina inclination assay. DS, HDS and 26-norCS shows approximately 20,20 and $80 \%$ less activity compared to that of CS, respectively.

sequence, DS, 26-norCS, and CS were identified as the products of HDS in P. vulgaris.

To establish the metabolic sequence of HDS, the metabolism of DS, 26-norCS, and CS were subsequently evaluated via the same enzyme assay. When DS was added to the enzyme solution as a substrate, CS and 26-norCS were detected as products by GC-MS analysis (Table 1). When CS was used, 26-norCS was identified as a product. When 26-norCS was added, no BRlike compounds were detected. The conversion rate of HDS to DS, DS to CS and CS to 26-norCS measured by GC-SIM calibration analysis was 3, 4 and 10\% in the enzyme assay, respectively. Collectively, the metabolic sequence, HDS $\rightarrow$ DS $\rightarrow$ $\mathrm{CS} \rightarrow$ 26-norCS, which is not reversibly converted, was confirmed in P. vulgaris.

We demonstrated via in vitro enzymatic conversion that CS can be generated from DS in $P$. vulgaris. ${ }^{6}$ This implies that two $\mathrm{C}_{28}$-BRs biosyntheses - namely 24-methylene and 24-methyl BRs biosynthesis - are biosynthetically connected, and can produce the physiologically important $\mathrm{BR}, \mathrm{CS}$, in plants. In this study, we also demonstrated that HDS can be converted to CS via $\mathrm{DS}$ in Phaseolus. Therefore, a $\mathrm{C}_{29}$-BRs biosynthesis, namely 24-ethylidene BRs biosynthesis, is also likely to be biosynthetically connected to the production of CS via DS in plants. In the tomato, 28-norCS, an end product of a C27-BRs biosynthesis, specifically 24-nor BRs biosynthesis, has been identified as a direct precursor of CS. ${ }^{7}$ Collectively, a variety of BRs biosyntheses are biosynthetically connected and maintain endogenous CS levels in plants. For this reason, endogenous CS levels are generally higher than those of other BRs in plants.

Biological activity, as assessed via rice lamina inclination tests, ${ }^{8}$ demonstrates that DS and HDS are less active than CS (Fig. 2). 26-norCS is also biologically less active than CS. Considering the metabolic sequence observed in the Phaseolus plants - HDS $\rightarrow$ DS $\rightarrow$ CS $\rightarrow$ 26-norCS, the demethylation of C-28 from HDS intermediated by DS is a biosynthetic reaction that increases BR activity, and the C-26 demethylation of CS to 26-norCS is a catabolic reaction that reduces the activity of an active $\mathrm{BR}, \mathrm{CS}$, in P. vulgaris. ${ }^{9}$

Acknowledgments. This study was supported by the ChungAng University Research Grants in 2010.

\section{References}

1. Bishop, G. J.; Yokota, T. Plant Cell Physiol. 2001, 42, 114.

2. Bajguz, A.; Tretyn, A. Phytochemistry 2003, 62, 1027.

3. Fujioka, S.; Yokota, T. Annu. Rev. Plant Biol. 2003, 54, 137.

4. Fujioka, S. In Brassinosteroids: Steroidal Plant Hormones; Sakurai, A., Yokota, T., Clouse, S. D., Eds.; Springer-Verlag: Tokyo, 1999; p 21.

5. Hwang, J.-Y.; Joo, S.-H.; Park, C. H.; Lee, S. C.; Kim, S.-K. Bull. Korean Chem. Soc. 2009, 30, 293.

6. Joo, S.-H.; Hwang, J.-Y.; Park, C. H.; Lee, S. C.; Kim, S.-K. Bull. Korean Chem. Soc. 2009, 30, 502.

7. Kim, T.-W.; Chang, S. C.; Lee, J. S.; Takatsuto, S.; Yokota, T.; Kim, S.-K. Plant Physiol. 2004, 135, 1231.

8. Wada, K.; Marumo, S.; Ikekawa, N.; Morisaki, M.; Mori, K. Plant Cell Physiol. 1981, 22, 323.

9. Kim, T.-W.; Park H.-H.; Kim, S. K. Bull. Korean Chem. Soc. 2004, 25,955 . 